\title{
Prognostic significance of infarct core pathology in ST-elevation myocardial infarction survivors revealed by non-contrast T1 mapping cardiac magnetic resonance
}

\author{
David Carrick ${ }^{3,1^{*}}$, Caroline Haig ${ }^{2}$, Samuli M Rauhalammi ${ }^{3}$, Nadeem Ahmed ${ }^{3}$, Ify Mordi ${ }^{3}$, Margaret McEntegart ${ }^{1}$, \\ Mark Petrie ${ }^{1}$, Hany Eteiba', Stuart Hood ${ }^{1}$, Stuart Watkins ${ }^{1}$, Mitchell Lindsay ${ }^{1}$, Ahmed Marous ${ }^{1}$, \\ Aleksandra Radjenovic ${ }^{3}$, Ian Ford ${ }^{2}$, Niko Tzemos ${ }^{3}$, Keith G Oldroyd ${ }^{3}$, Colin Berry ${ }^{3}$
}

From 18th Annual SCMR Scientific Sessions

Nice, France. 4-7 February 2015

\section{Background \\ Myocardial longitudinal relaxation time (T1, ms) is a fundamental magnetic property of tissue that is related to water content and mobility. The pathophysiological and prognostic importance of native myocardial $\mathrm{T} 1$ values in acute ST-elevation myocardial infarction (STEMI) patients is unknown. We aimed to assess the clinical significance of infarct core native $\mathrm{T} 1$.}

\section{Methods \\ We performed a prospective single center cohort study in reperfused STEMI patients who underwent CMR 2 days and 6 months post-MI. Native T1 CMR (MOLLI investigational prototype sequence: 3 (3) 3 (3) 5) was measured in myocardial regions-of-interest. The infarct territory and microvascular obstruction (MVO) were depicted with late gadolinium enhancement CMR. Adverse remodeling was defined as an increase in $\mathrm{LV}$ end-diastolic volume (LVEDV) $\geq 20 \%$ at 6 months. All- cause death or heart failure hospitalization was a pre- specified outcome that was assessed during follow-up.}

\section{Results}

300 STEMI patients (mean \pm SD age $59 \pm 12$ years, $74 \%$ male, 114 with anterior STEMI) gave informed consent and had CMR (14 July 2011 - 22 November 2012). Of these, 288 STEMI patients had evaluable T1 maps. Infarct

${ }^{3}$ Institute of Cardiovascular \& Medical Sciences, University of Glasgow, Glasgow, UK

Full list of author information is available at the end of the article size was $18 \pm 14 \%$ of LV mass. One hundred and forty five (50\%) of 288 patients had late MVO, whereas 160 (56\%) patients had infarct core pathology revealed by native $\mathrm{T} 1$. Native T1 within the infarct core $(996.9 \pm 57.3 ; \mathrm{p}<0.01)$ was higher than in the remote zone $(961 \pm 25 \mathrm{~ms} ; \mathrm{p}<0.01)$ but lower than in the area-at-risk (1097 $\pm 52 \mathrm{~ms})$. In multivariable linear regression, native $\mathrm{T} 1$ in the infarct core was negatively associated with age, initial systolic blood pressure, TIMI coronary flow grade at initial angiography, Killip class at presentation and neutrophil count (all $\mathrm{p}<0.05)$, independent of LVEF, LVEDV or infarct size.

At 6 months, LVEDV increased by $5(25) \mathrm{ml}(\mathrm{n}=262$ patients with evaluable data). Adverse remodeling occurred in $30(12 \%)$ patients and $23(76.7 \%)$ of these patients MVO at baseline. T1 in the infarct core was a multivariable predictor of adverse remodeling $(-0.01$ $(-0.02,-0.00) ; \mathrm{p}=0.048)$.

$288(100 \%)$ patients were followed-up for a median of 845 days. Thirty $(10.4 \%)$ patients died or experienced a heart failure event and 13 (4.5\%) of these patients experienced the event post-discharge. Infarct core native T1 predicted all-cause death or heart failure post-discharge (hazard ratio $0.969,95 \%$ CI $0.953,0.985 ; \mathrm{p}<0.001$ ) including after adjustment for LVEF $(\mathrm{p}<0.001)$ and LVEDV at baseline $(\mathrm{p}<0.001)$, and was comparable with MVO.

\section{Conclusions}

Infarct core native $\mathrm{T} 1$ represents a novel alternative non-contrast imaging biomarker for infarct characterization and prognostication in STEMI survivors. 


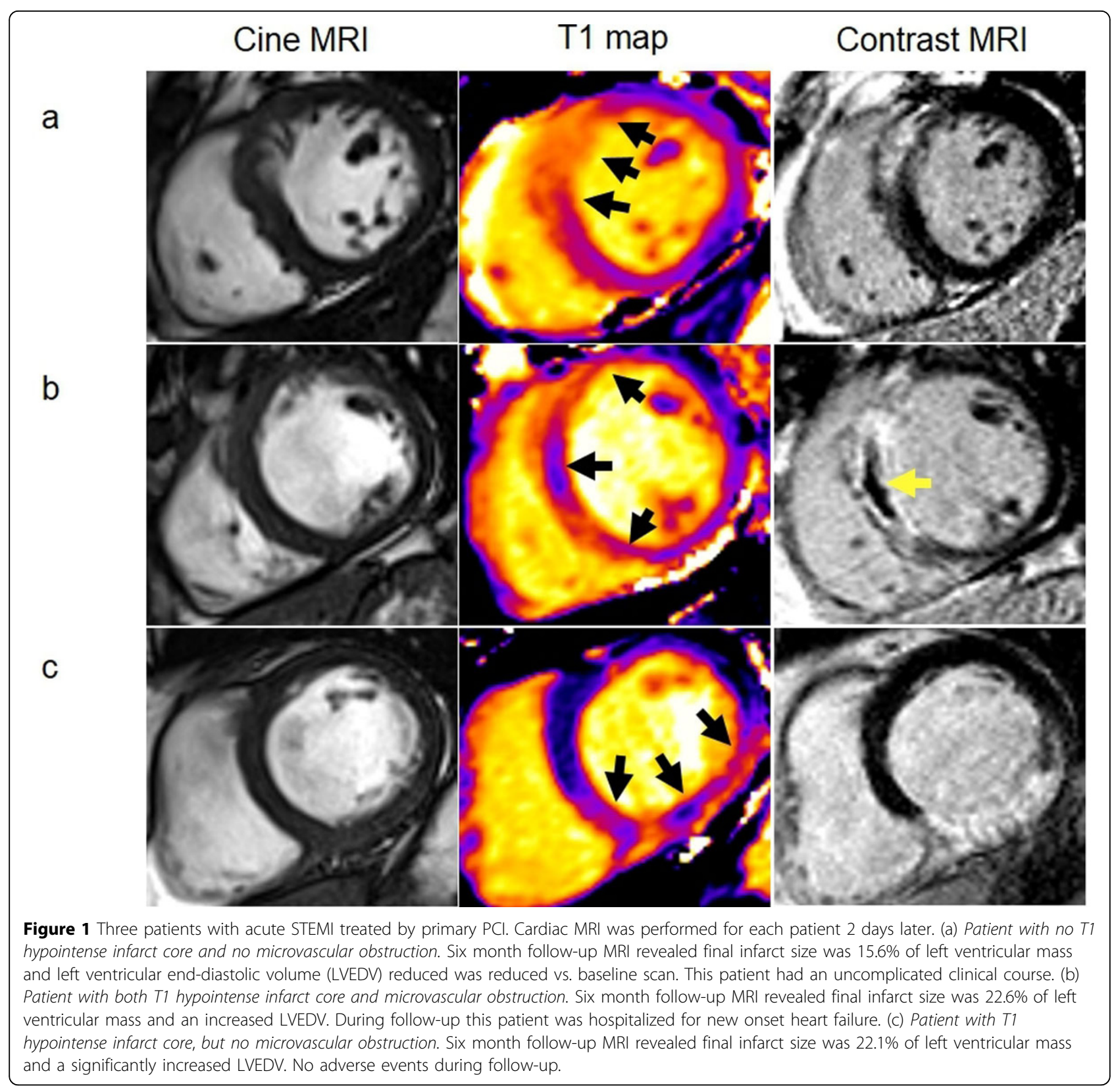

\section{Funding}

N/A.

\section{Authors' details}

'Golden Jubilee National Hospital, Clydebank, UK. ${ }^{2}$ Robertson Center for Biostatistics, University of Glasgow, Glasgow, UK. ${ }^{3}$ Institute of Cardiovascular \& Medical Sciences, University of Glasgow, Glasgow, UK.
doi:10.1186/1532-429X-17-S1-026

Cite this article as: Carrick et al:: Prognostic significance of infarct core pathology in ST-elevation myocardial infarction survivors revealed by non-contrast T1 mapping cardiac magnetic resonance. Journal of Cardiovascular Magnetic Resonance 2015 17(Suppl 1):O26.

Published: 3 February 2015 